\title{
LibQA - Library Quality Assurance for VHDL Synthesis and Simulation
}

\author{
Ronald B. Stewart, SGS THOMSON Microelectronics
}

Jon Phillips, Philips Semiconductors

\begin{abstract}
LibQA is a quality assurance tool for VHDL synthesis and simulation models which also performs timing characterization. The synthesis model is translated into an FSM, then graph exploration generates stimuli for VHDL and electrical simulation. Function, propagation delay, timing constraint violation, and hazard response are all tested.
\end{abstract}

\section{Introduction}

Correct standard cell models are a good foundation for successful synthesis and simulation. LibQA is a qualityassurance tool currently in industrial service to compare Synopsys synthesis models against VHDL simulation models and against electrical models extracted from layout. Starting from a synthesis model, LibQA generates an equivalent FSM. Using graph algorithms, LibQA generates stimuli for simulations. The results of the simulations give these benefits:

- Synopsys Technology Library cell model testing

- VHDL cell model testing

- Cell timing characterization

In most cases, LibQA is automatic. However, cells which cannot be defined in the syntax of the synthesis model require manually supplied stimuli.

The synthesis model is analyzed formally, and the simulation stimuli generated from it are formally correct. Assuming there are no unexpected states in the models, the test coverage is total, showing that the electrical and VHDL models implement the specification of the synthesis model. Others have proven equivalence of certain VHDL models using formal methods, but this is not practical for a general production tool. Abstraction of electrical models has been done so far without proof, and is limited in scope. Progress on those two fronts should create new opportunities in model validation.

LibQA also supports inspection of timing models:

- Cell timing range and monotonicity checks

- Cell timing graph viewer

- Cell timing calculator

\section{FSM Construction}

LibQA translates the Synopsys Technology Library synthesis model into a Mealy machine[1] $\mathbf{M}=(\mathbf{Q}, \Sigma, \Delta, \delta, \lambda$, $\mathrm{q}_{0}$ ) where $\mathbf{Q}=\left\{\mathrm{q}_{0}, \mathrm{q}_{1}, \cdots \mathrm{q}_{\mathrm{n}}\right\}$ is the set of states, $\Sigma=\left\{\mathrm{a}_{0}\right.$, $\left.a_{1}, \ldots a_{n}\right\}$ is the input alphabet, $\Delta=\left\{a_{0}, a_{1}, \ldots a_{n}\right\}$ is the output alphabet, $\delta$ is the state transition function mapping $\mathbf{Q} \times \Sigma$ to $\mathbf{Q}, \lambda$ is the output function mapping $\mathbf{Q}$ to $\Delta$, and $\mathrm{q}_{0}$ is the initial state.

For this application, some preliminary definitions relate the abstract machine to the physical cell model. Define $\mathrm{V}$ $=\{0,1, Z\}$ the set of signal values, $v \in V$, and $I=\left\{i_{0}, i_{1}, \cdots\right.$ $\left.\mathrm{i}_{\mathrm{n}}\right\} \subset \mathrm{V}^{\mathrm{n}}$ the set of inputs. The single input transition function $I_{n+1}=f\left(I_{n}, i, v\right)$ produces a new input combination from an old one, so that $i_{j+1}=i_{j}, j \neq n$, and $i_{n}=v$.

$\Sigma$ is defined as a set of all one-signal input signal transitions (i,v), $\mathbf{Q}$ is a set of input signal and state variable current values, and $\Delta$ is a set of output signals defined in $\{0$, $1, \mathrm{Z}\} . \mathrm{q}_{0}$ is defined for each test set so that values on primary inputs are assigned to the associated $\mathrm{q}_{\mathrm{i}}$, and values taken from the state variables of Synopsys sequential primitives ff and latch are assigned ' $U$ '. These conventions are well suited to the waveform generation task needed to run the simulators.

\section{FSM for Combinational Cells}

To benefit from the simple graph search algorithms that generate the test vectors, even the combinational cells are translated into FSMs. To illustrate the process, here is the functional part of the synthesis specification for a tristate buffer with resistive input pull-up:

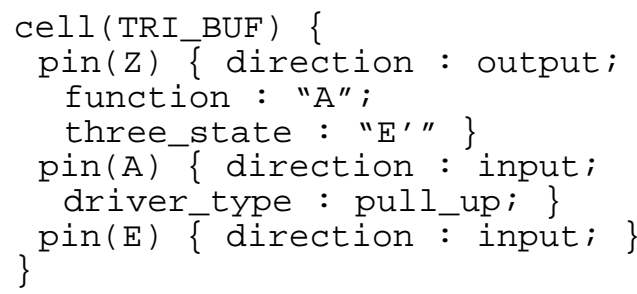

The steps are:

1. Define the set of states. There is one state variable for each input. Variables are Boolean unless the 
driver_type attribute is present, in which case such variables are on $\{0,1, \mathrm{Z}\}$.

Given input signals $\mathrm{A}$ and $\mathrm{E}, \mathbf{Q}=\mathrm{A} \times \mathrm{E}$ is the set of states, where $A \in\{0,1\}$ and $Z \in\{0,1, Z\}$. For readability, label them $\mathrm{qAE}=\{\mathrm{q} 00, \mathrm{q} 01, \mathrm{q} 0 \mathrm{Z}, \ldots\}$

2. Define the input alphabet as the set of all signal transitions on individual signals. For a Boolean input signal, the transitions are $\{01,10\}$. For signals on $\{0,1, Z\}$ the transitions are $\{01,0 \mathrm{Z}, 10,1 \mathrm{Z}, \mathrm{Z} 1, \mathrm{Z} 0\}$.

Thus, label the input alphabet $\Delta=\{\mathrm{e} 01, \mathrm{e} 10, \mathrm{a} 01, \mathrm{a} 0 \mathrm{Z}$, a10, a1Z, aZ1, aZ0\}.

3. Define the state transition function. $I_{n+1}=f\left(I_{n}, i, v\right)$.

The table contents are generated by evaluating the definition over its range.

Table 1: $\delta$ for TRI_BUF

\begin{tabular}{|l|l|l|l|l|l|l|l|l|}
\hline & $e 01$ & $e 10$ & $a 01$ & $a 0 Z$ & $a 10$ & $a 1 Z$ & $a Z 1$ & $a Z 0$ \\
\hline \hline$q 00$ & $q 10$ & - & $q 01$ & $q 0 Z$ & - & - & - & - \\
\hline$q 01$ & $q 11$ & - & $q 11$ & - & $q 00$ & $q 0 Z$ & - & - \\
\hline$q 0 Z$ & $q 1 Z$ & - & $q 1 Z$ & - & - & - & $q 01$ & $q 00$ \\
\hline$q 10$ & - & $q 00$ & - & $q 1 Z$ & - & - & - & - \\
\hline$q 11$ & - & $q 01$ & - & - & $q 10$ & $q 1 Z$ & - & - \\
\hline$q 1 Z$ & - & $q 0 Z$ & - & - & - & - & $q 11$ & $q 10$ \\
\hline
\end{tabular}

4. Define the output alphabet. This is the set of values for the output pins. Each occurrence of pin () \{ direction:output $\}$ is an output. The values are normally Boolean, but they become $\{0,1, \mathrm{Z}\}$ if the record three_state is present.

There is one three_state output, Z. The output alphabet is $\Delta=\{0,1, \mathrm{Z}\}$.

5. Define the output function mapping $\mathbf{Q}$ to $\Delta$. For each output, evaluate the function and three_state expressions. If three_state is present and evaluates as true, the output value is $\mathrm{Z}$; otherwise, the output is the value of the function expression. The independent variables of the two expressions correspond to the state variables, but the range of the expressions is Boolean, while that of the state variables is $\{0,1, \mathrm{Z}\}$. Because $\mathrm{Z}$ is caused only by the driver_type attribute, a Boolean value is obtained for every occurrence of Z: pull_up maps Z to
1, and pull_down maps $\mathrm{Z}$ to 0 . The evaluation then proceeds by converting the infix to a postfix tree and evaluating from leaf to summit. $\lambda$ is the output function mapping $\mathbf{Q}$ to $\Delta$.

Table 2: $\lambda$ for TRI_BUF

\begin{tabular}{|l||l|l|l|l|l|l|}
\hline State $q A E$ & $q 00$ & $q 01$ & $q 0 Z$ & $q 10$ & $q 11$ & $a 1 Z$ \\
\hline \hline Output Z & 1 & 0 & 0 & $Z$ & $Z$ & $Z$ \\
\hline
\end{tabular}

Not surprisingly, the table turns out to be simply the truth table for the combinational cell. All the other machinery has been created to give these conveniences:

- The data structures are identical to those to be used for sequential cells, supporting the graph algorithms used to generate test stimuli.

- Only single input transitions exist, supporting the definition and application of timing events.

\section{Sequential Cell FSM Construction}

Synopsys models sequential cells use just two primitives: ff for flip-flops, and latch for latches. There is also an obsolete state primitive we do not use.

Here is a Synopsys description of a sample flip-flop:

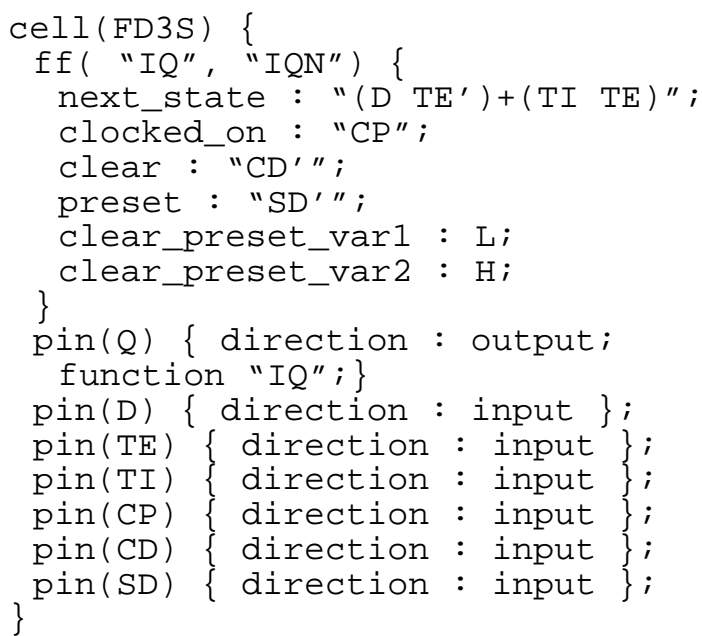

The steps LibQA uses to construct its FSM model are:

1. Call construct_ff, which returns an FSM where boolean expressions next_state, clocked_on, and all the other ff inputs are represented by a single term.

2. Expand the FSM for each ff input expression. 
3. Construct the output function $\Delta$.

FSM * construct_ff( ) is a function which constructs a complete FSM structure which models an elementary ff. These come in several variations depending on attributes which concern the preset and clear functions:

- whether preset and clear exist.

- the state when clear and set are applied together.

For example, a multiplexed D ff is expressed as:

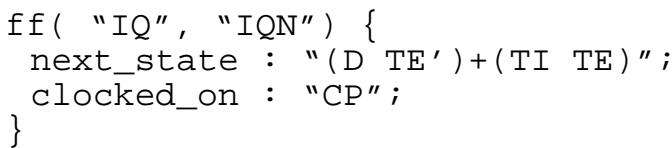

The FSM will be constructed by first calling construct_ff to return an elementary FSM for the ff. Then, new state variables are created for each input, and each arc to the (now replaced) next_state and clocked_on variables is replaced by a set of arcs accounting for all the cases due to the expressions. In the present case, clocked_on is simple, so re-labeling is all that is needed.

The ff primitive is expanded to:

Table 3: $\delta$ for simplest ff

\begin{tabular}{|l|l|l|l|l|}
\hline $\begin{array}{c}(I Q, \\
\text { next, } \\
\text { clock })\end{array}$ & $\begin{array}{c}\text { next } \\
\text { state } \\
\text { Rise }\end{array}$ & $\begin{array}{c}\text { next } \\
\text { state } \\
\text { Fall }\end{array}$ & $\begin{array}{c}\text { clock } \\
\text { Rise }\end{array}$ & $\begin{array}{c}\text { clock } \\
\text { Fall }\end{array}$ \\
\hline \hline$(0,0,0)$ & $(0,1,0)$ & - & $(0,0,1)$ & - \\
\hline$(0.0,1)$ & $(0,1,1)$ & - & - & $(0,0,0)$ \\
\hline$(0,1,0)$ & - & $(0,0,0)$ & $(1,1,1)$ & - \\
\hline$(0,1,1)$ & - & $(0,0,1)$ & - & $(0,1,0)$ \\
\hline$(1,0,0)$ & $(1,1,0)$ & - & $(0,0,1)$ & - \\
\hline$(1,0,1)$ & $(1,1,1)$ & - & - & $(1,0,0)$ \\
\hline$(1,1,0)$ & - & $(1,1,0)$ & $(1,1,1)$ & - \\
\hline$(1,1,1)$ & - & & - & $(1,1,0)$ \\
\hline$(U, 0,0)$ & $(U, 1,0)$ & - & $(0,0,1)$ & - \\
\hline$(U, 0,1)$ & $(U, 1,1)$ & - & - & $(U, 0,0)$ \\
\hline$(U, 1,0)$ & - & $(U, 0,0)$ & $(1,1,1)$ & - \\
\hline$(U, 1,1)$ & - & $(U, 0,1)$ & - & $(U, 1,0)$ \\
\hline
\end{tabular}

\section{Test Sequence Generation}

The different types of test sequence LibQA can make are:

- Functional exercise

- Timing exercise

- Timing constraint violation

- Hazard exercise

The $\mathrm{C}$ procedures which implement them are described below. Each returns a linked list of path data structures:

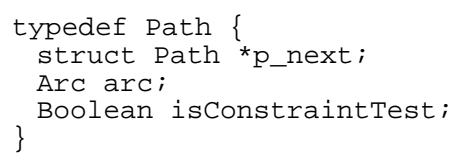

\subsection{FindPath Procedure}

This is a depth-first path enumeration. The GoodArc and Done functions are assigned to suit the kind of path needed: state-to-state, all arcs, or all timing arcs. The complexity of standard-cell FSM models is low, so performance is acceptable. The search algorithm is elementary:

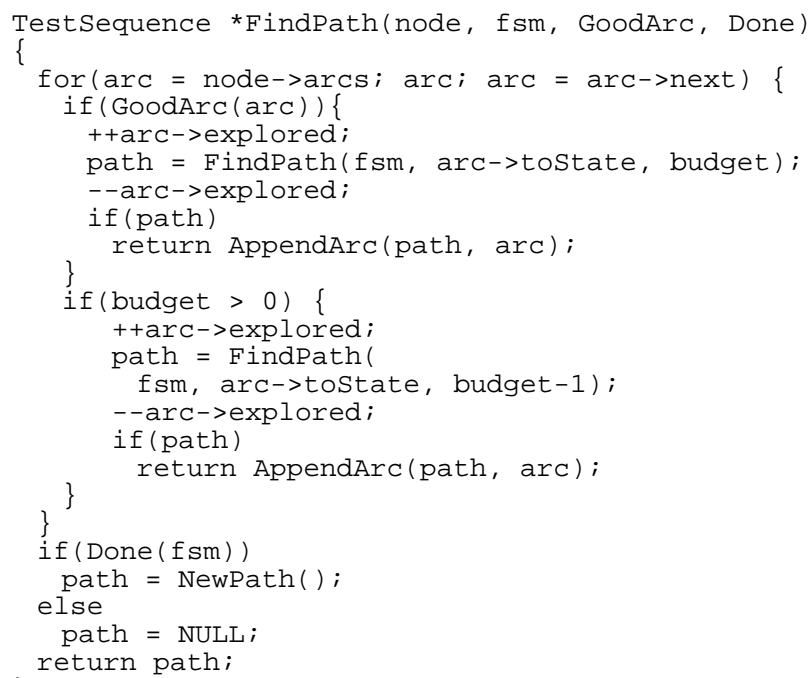

\subsection{FindTest Procedure}

A test sequence is selected so that every arc of the FSM is traversed in optimal or near-optimal order. Optimal order is not useful when finding it costs more than the improvement in simulation time it buys, so sub-optimal paths are accepted when complexity exceeds a limit.

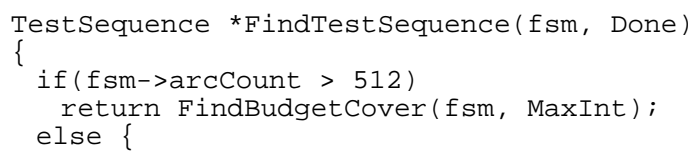




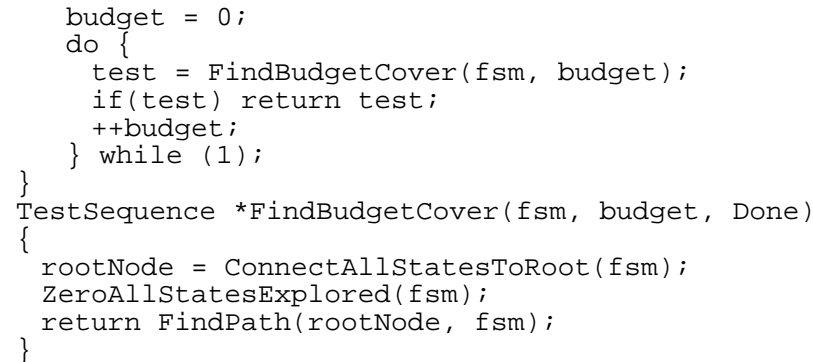

\subsection{Functional Test Sequence}

The functional test sequence traverses every arc of the FSM at least once.

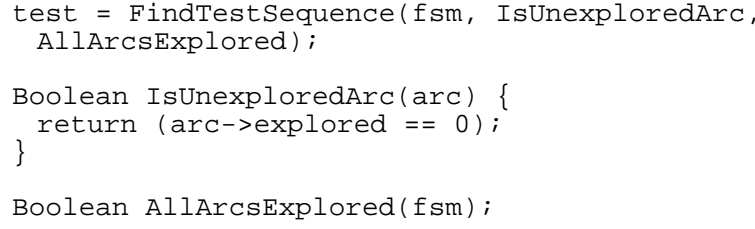

\subsection{Timing Test Sequence}

Only the arcs that result in an output signal change are required in the timing test sequence. The resulting sequence is much shorter than the functional test.

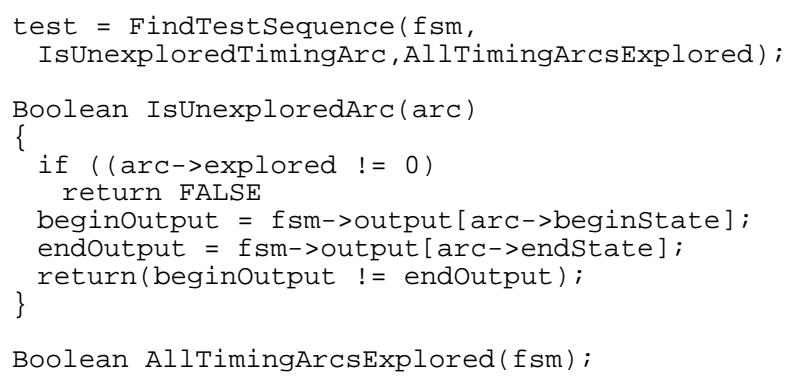

\subsection{Violation Test Sequence}

Knowledge of the state of a cell can be lost if a timing constraint is violated. A set of classically recognized constraint violations is produced for the latch and ff primitives, suitably expanded according to their associated expressions. No attempt was made to inspect the FSM trying to recognize these, but this a good opportunity for future improvement.

The catalog of constraints is:

- Minimum Pulse Width. All terms of clocked_on, clear, and preset expressions are exercised for minimum pulse width.

- Setup and Hold. All terms of next_state are exercised for setup and hold.
- Recovery. All terms of preset and clear are exercised for recovery time in combination with all terms of clocked_on.

\subsection{Hazard Test Sequence}

VHDL models can be configured to respond to hazards in a variety of ways: with transport delays, inertial delays, glitch $\mathrm{X}$ generation, spike $\mathrm{X}$ generation, and message generation. All arcs leading to an output change can be subjected to hazards. Testbenches are generated for all these behaviors because all possible choices need to be tested.

\section{Electrical Testbench Generation}

For functional testing, an Eldo[4] electrical simulation file is generated from the functional stimuli and the environmental information in the synthesis model. The simulation is carried out, and the output values are captured for every input change. A defect is reported if any output differs from the predicted value. If there are hidden states, or electrical problems, this test may not notice them. The timing characterization phase, where a range of operating conditions is applied, can help to spot more subtle problems.

\section{VHDL Testbench Generation}

The properties of the VHDL models which need to be tested include functional operation, backannotated timing, $\mathrm{X}$ and report generation in response to glitches and timing constraint violations. The VHDL testbenches themselves consist of stimuli and procedures. We need to know if the models survive the stimuli without fatal errors, if the functional operation is correct, and if the timing values from the SDF are respected.

\subsection{VHDL Procedures}

\subsection{1 qa_input}

Apply STIMULUS_VALUE to the cell.

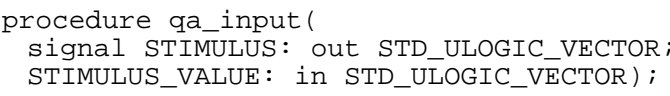

\subsection{2 qa_function}

This procedure verifies the function only - not the timing.

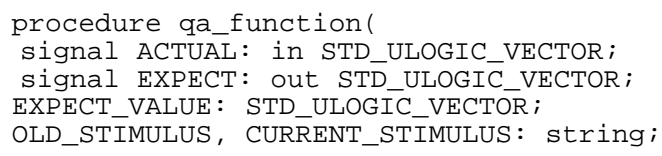


DELAY: time);

\subsection{3 qa_timing1}

This procedure verifies function and timing for a singleoutput cell.

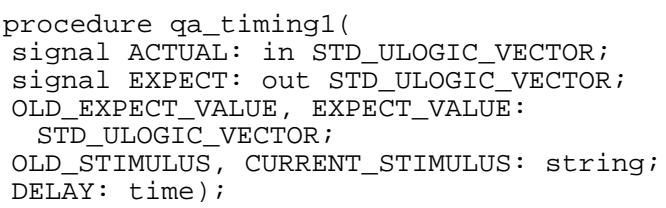

An excerpt shows how functional and timing errors are detected:

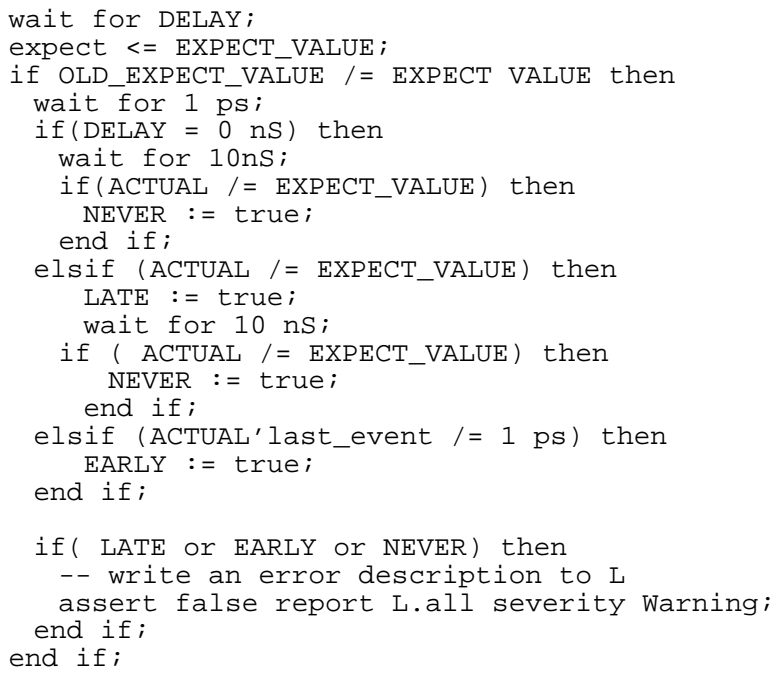

\subsection{4 qa_timing2}

Verify function and timing for a two-output cell. The code is a development of the method of qa_timing1. All functional and timing errors on both signals are reported.

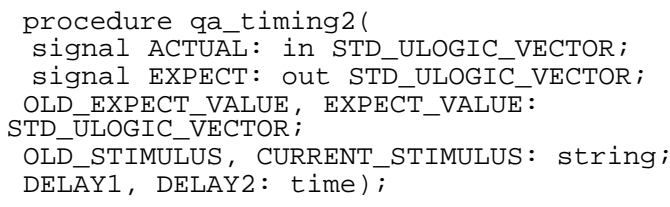

\subsection{VHDL Stimuli}

VHDL header and closing statements are generated from the FSM structure. The test sequence generation procedures return a path data structure for each kind of test: function, timing, violation, and glitch. Each of these is translated into VHDL stimuli. LibQA generates the constants that provide delay values for the stimuli and the constants for the SDF, the test benches can detect timing errors in the models. This is an excerpt from the timing test for an inverter; other tests follow similar lines.

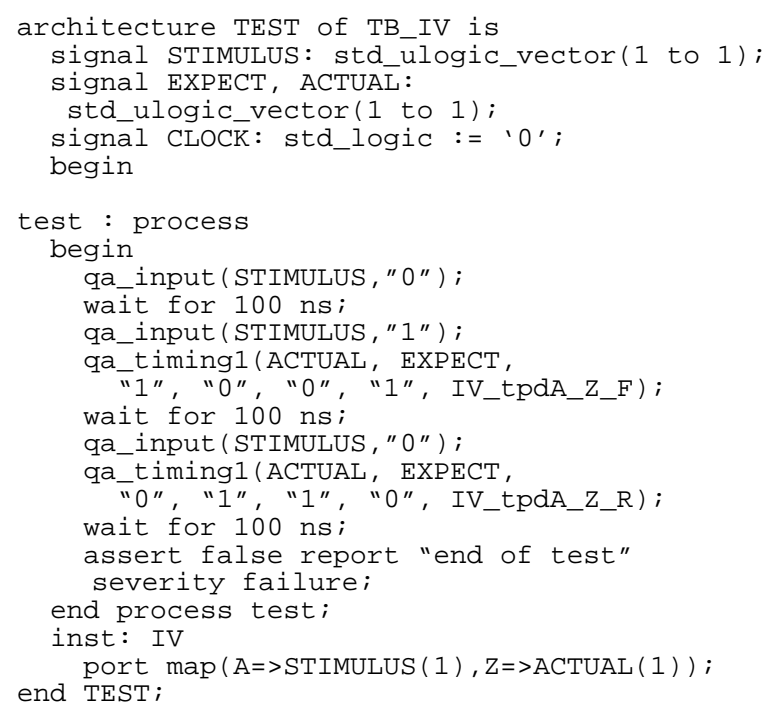

\section{Timing Characterization}

LibQA starts with an existing synthesis model, generates test patterns, runs electrical simulations, and produces a characterization database. Another program, U2STF [3], generates the new synthesis model set from the characterization database. A separate synthesis model is necessary for each operating condition (process, temperature, voltage) to avoid derating errors.

Propagation delays are measured with the timing test patterns previously described. The Eldo electrical simulator has primitive functions to do the measurements. One simulation is done for each point in the timing table. Both propagation delay and rise time are measured for all timing events in each run, while load capacitance and input rise time are parameters which change for each run. One Eldo description used for an inverter is:

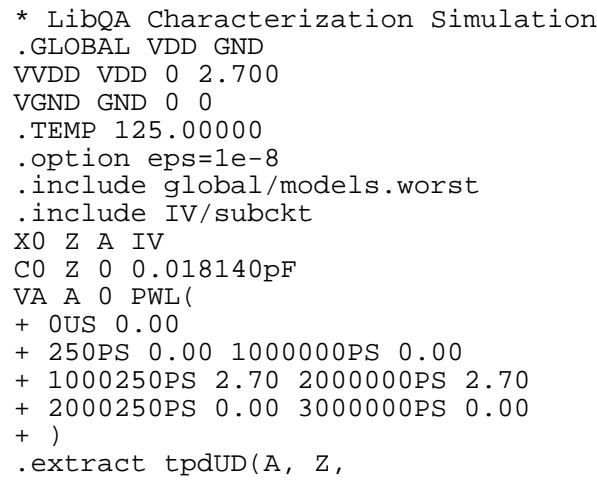




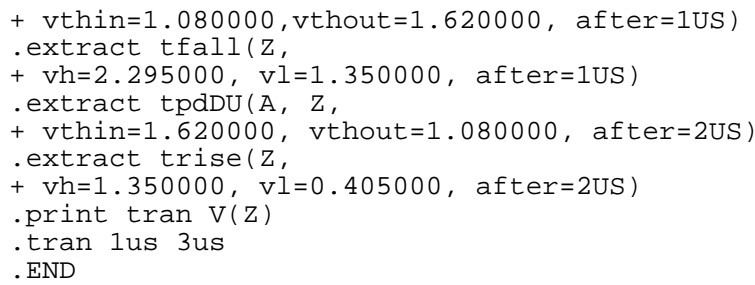

Often, a set of related events have negligible differences in propagation delay. LibQA groups these into equivalence classes. Where necessary, the classes are differentiated using sdf_cond attributes. This ability to decide which timing events to use gives an important contribution to accuracy.

Timing violations are measured with the violation test patterns previously described. A violation test tells whether the observable outputs failed to arrive at the expected value. A binary search on pass/fail gives a numerical result, but at more expense than the simpler delay measurements. Because each simulation run tests all the constraints, a separate binary search record is maintained for each one.

\section{Timing Report Generation}

LibQA tests the characterization database rather than the completed synthesis model because individual derating information is merged into global derating $\mathrm{k}$ _factors, so good scrutiny is not possible there. LibQA checks for properties that experience has linked to characterization problems. Statistics for each class of event are calculated, and individual events are compared to the statistics. These statistics are also used during synthesis model regeneration to calculate the $\mathrm{k} \_$factors. Some specific tests are:

- Monotonicity. Timing functions are tested for monotonicity with respect to all of their independent variables: input rise time, load, temperature, voltage, and process.

- Range. Check the range and domain of all timing functions. No timing should ever be negative, non-numeric, or infinite.

- Extreme values. For each class of event, the ten extreme cases are reported in ranked order. This can be interesting reading.

\section{Timing Graph Viewer and Calculator}

The timing graph viewer reveals timing properties difficult to recognize in tabular data. Timing characterization can produce subtle problems, but looking at every timing graph for a library will often reveal defects. The graphs can be output in printable format.

LibQA's calculator gives a numerical evaluation for any timing in the library.

\section{Results}

LibQA was used to develop a 151-cell library of 0.5 micron CMOS standard cells. Two of the cells ( buskeepers) required hand-generated stimuli because they had the 'dont_use' property and no functional description. Using a Sparc 10, functional verification using Eldo took 72 minutes, and some flip-flops having set and clear inputs were found incorrect when set and clear are applied together. Characterization for the library took 49 hours. As a result of the timing report, some of the max_cap values were corrected, and the choice of transition time and net_capacitance sample points were optimized. The VHDL simulation took 130 minutes, and an array out of bounds error due to a hand edited cell, and a number of undetected violations were revealed. The development time for LibQA was less than the time previously spent preparing tests, the coverage was much higher, and new classes of testing were applied. Finally, the time-to market for our latest library was significantly faster than the previous generation, and there have been no bug reports on the library.

\section{Conclusions}

LibQA is an industrial tool used to support the production and test of VHDL synthesis and simulation models. The application of formal methods increases precision and lowers costs. LibQA requires manual stimuli for cells which cannot be expressed in the syntax of the synthesis models, but it is fully automatic for 147 of 151 cells in a 0.5 micron CMOS standard cell library.

\section{References}

[1] "Introduction to Automata Theory, Languages, and Computation", Hopcroft and Ullman, Addison-Wesley Publishing Co., 1979

[2] "Library Compiler Reference Manual" Synopsys, Inc., March 1994

[3] "U2STF User Guide", SGS-Thomson Microelectronics, November 1994

[4] "ELDO Electrical Circuit Simulator”, Anacad, November 1994 